\title{
Corrélation entre les notations en serre et au champ de la résistance de lignées de maïs envers le virus de la mosaïque nanisante du maïs (MDMV-A)
}

\author{
B Alliot ${ }^{1 *}, \mathrm{E}$ Gellez ${ }^{2}$, PA Signoret ${ }^{1}$ \\ 1 ENSA-INRA, UFR de biologie et pathologie végétales, 2, place Pierre-Viala, F34060 Montpellier cedex 1; \\ 2 Maïsadour, BP 27, F40001 Mont-de-Marsan cedex
}

(Reçu le 20 mai 1995 ; accepté le 4 juillet 1995)

\begin{abstract}
Résumé - Le virus de la mosaïque nanisante du maïs souche A (maize dwarf mosaic virus = MDMV-A) est présent dans tous les pays du bassin méditerranéen. Ce virus étant transmis par pucerons selon le mode non-persistant, la lutte chimique est inefficace ; il faut donc rechercher des lignées résistantes. En effet, le MDMV, qui affecte de façon significative les hybrides, se révèle plus agressif sur les lignées et sera donc préjudiciable en production de semences. Pour étudier la résistance du matériel génétique utilisé en Europe, il a fallu répertorier les souches de MDMV-A. Une collaboration entre I'ENSA-INRA de Montpellier et la société Maïsadour a consisté, dans un premier temps, à prospecter et à caractériser les différentes isolats de la zone méditerranéenne. Ceux-ci ont pu être classés suivant le poids moléculaire de leur capside protéique (Signoret et al, 1993). Des inoculations à partir des 2 souches les plus représentatives ont ensuite été réalisées en enceintes climatisées et au champ. Les essais en conditions contrôlés ont été faits et les résultats interprétés suivant la méthode du «disease index »(Kuhn et Smith, 1977). Au champ, 10 notations ont été effectuées. Celles-ci concernent des caractères quantitatifs facilement mesurables pendant le cycle de la plante. Une corrélation a pu être mise en évidence entre le disease index et l'importance de la maladie chez des 650 lignées inoculées au champ.
\end{abstract}

Zea mays = maïs / virus de la mosaïque nanisante du maïs / MDMV-A / disease index / hérédité de la résistance

Summary - Correlation between greenhouse and field scores in testing resistance of inbred maize lines to maize dwarf mosaic virus-A (MDMV-A). In the Mediterranean countries, maize can be infected by several viruses, the most prevalent of which is maize dwarf mosaic virus strain A (MDMV-A). This potyvirus is non-persistently transmitted by several aphids and cannot be controlled by chemical insecticides. The reservoir host in the fields, Johnson grass (Sorghum halepense), is very common. Its control by herbicides is possible but not always feasible. In commercial hybrid maize fields, the incidence of MDMV is not very important but, in seed production inbred lines are severely affected and seed yield is very often decreased. The only method of control would be to release resistant inbred lines. In order to check the germplasm used in Europe we first started a study on the biodiversity of MDMV-A. The results obtained are presented in another paper; we can differentiate MDMV isolates according to the molecular weight of the capsid and the reaction of inoculated inbred lines. Mechanical inoculations of our germplasm using an artist's airbrush have been carried out in climatic chambers and in the open field. To evaluate corn for resistance to MDMV-A in controlled conditions, we have used the disease index system proposed by Kuhn and Smith (1977) based on the percentage of plants developing symptoms and the date of symptom appearance after inoculation. In the field, we have made 10 observations concerning easily quantifiable characteristics throughout the growing period of maize. The index values were correlated to the incidence of disease (expressed as quantitative characteristics) for 650 inbred lines inoculated in the field.

Zea mays = maize / maize dwarf mosaic virus / disease index / inheritance of resistance

\footnotetext{
* Correspondance et tirés à part
} 


\section{MATÉRIELS ET MÉTHODES}

Des lignées de maïs considérées comme résistantes, aux États-Unis, au virus de la mosaïque nanisante du maïs souche A (MDMV-A), se sont révélées sensibles à ce même virus dans les conditions du bassin méditerranéen. Partant de cette observation, nous avons réalisé une prospection dans les zones de culture, plus particulièrement dans les zones de production de semences, en Espagne, dans le sud-est de la France et en Italie du Nord, afin de comparer les isolats présents.

Les isolats ont pu être classés en différents groupes selon le poids moléculaire de leur capside protéique. L'isolat nommé PER (de la localité Perpignan, France) est le plus fréquent, l'isolat appelé LAV (Lavalette, France) se révèle être le plus agressif. Aucun pied de maïs ou de sorgho d'Alep infesté ne l'était par la souche communément décrite aux États-Unis, souche MDMV-A-USA (Signoret et al, 1993).

Depuis 1987, une collection de lignées de maïs est implantée dans l'une des stations de sélection de Maïsadour (Fourques, France) dans le but d'y étudier le comportement vis-à-vis du MDMV-A. Trois répétitions de 56 plantes de chaque lignée sont inoculées au stade 3-4 feuilles à l'aide d'un aérographe, à une pression de $8 \mathrm{~kg}$. Les inoculations ont été réalisées avec l'isolat LAV dont les symptômes sont plus faciles à déceler. L'inoculum est préparé par broyage des feuilles dans un tampon phosphate $0,01 \mathrm{M} \mathrm{pH} \mathrm{7,5} \mathrm{à}$ raison de $1 \mathrm{~g}$ de feuille pour $9 \mathrm{ml}$ de tampon; $75 \mathrm{mg} / \mathrm{ml}$ de carborundum 200 mesh sont ajoutés comme abrasif. Un second bloc, comprenant également 3 répétitions de 56 plantes par lignée, sert de témoin. Dix notations sont effectuées à différents moments de la période végétative et reproductive de la plante. Les notations suivantes sont considérées, pour notre interprétation future, comme des valeurs quantitatives :

i) pourcentage de plantes présentant des déficiences chlorophyliennes 3 sem après l'inoculation ;

ii) hauteur des plantes inoculées en comparaison avec les témoins, notation effectuée pour chaque lignée à la fin de la floraison mâle ;

iii) longueur de l'épi principal (\% par rapport au témoin) ;

iv) diamètre de l'épi ;

v) nombre de rangs de l'épi ;

vi) nombre de grains par rang ;

vii) poids de 1000 grains à 14,5\% d'humidité ;

viii) poids spécifique;

ix) humidité du grain à la récolte ;

x) rendement à la récolte.

Les 8 dernières notations sont toutes réalisées au moment de la récolte.

Ce travail, qui a débuté il y a 8 ans, nous a permis de constituer un fichier riche de 650 lignées. Notre souci a été par la suite de pouvoir interpréter cette quantité d'information en évitant au maximum le biais qu'apporte toujours une expérimentation au champ. La précision qui nous paraissait indispensable était de $8 \%$ de coefficient de variation en ce qui concerne le rendement, et de $2 \%$ pour l'humidité.

Des lots de 4 fois 25 plantes, de lignées dont nous avions observé le comportement au champ depuis plusieurs années, plants âgées de 9 j, ont été inoculés en enceintes climatisées. Des notations de présence de symptômes visibles ont été faites après $6,11,16$ et 28 j d'inoculation. Un coefficient de 4 à 1 a été attribué aux différents relevés (4 pour le $\%$ de plantes présentant des symptômes au stade 6 j (W), 3 pour le \% de plantes présentant des symptômes au stade $11 \mathrm{j}(\mathrm{X}), 2$ pour la notation au $16^{\mathrm{e}}$ jour $(\mathrm{Y})$ et 1 pour le relevé ultime après $28 \mathrm{j}(Z))$. Un index de maladie "disease index", a été calculé ainsi : $4 W+3 X+2 Y+Z$. Les valeurs, comprises entre 0 et 1000 permettent de regrouper les lignées en 3 classes afin de juger de leur comportement au virus : 0 à 350 résistant, 351 à 650 intermédiaire, 651 à 1000 sensible (Kuhn et Smith, 1977).

\section{RÉSULTATS}

\section{En enceintes climatisées}

Le tableau I regroupe les résultats obtenus en enceintes climatisées par la méthode du disease index. Il montre que la cinétique de l'extériorisation des symptômes est différente suivant les lignées et les souches utilisées.

Nous pouvons ainsi classer ces 3 lignées visà-vis du MDMV-A, LFF07 : en hautement résistante, FPLATA : résistante : et LFF12 : sensible.

\section{Au champ}

Les notations au champ fournissent des résultats plus ou moins intéressants, la moyenne (MOY) des résultats obtenus sur 650 lignes, en ce qui concerne les différents caractères analysés, est indiquée ci-dessous :

- le \% de plantes présentant des symptômes $(-33,8 \%)^{\star *}$;

- la différence de hauteur entre témoin et virosé $(-2,87 \%)^{* *}$

- la diminution de la longueur de l'épi $(-0,75 \%)$ NS ;

- la diminution du diamètre de l'épi $(-0,50 \%)$ NS ;

- la diminution du nombre de rang de l'épi $(-1,10 \%)$ NS ;

- la diminution du nombre de grain par rang $(-0,90 \%)$ NS ; 
Tableau I. Résultats des inoculations par les 2 isolats de MDMV-A utilisés pour le calcul du disease index (pourcentage de plantes malades aux différentes dates de notation).

\begin{tabular}{|c|c|c|c|c|c|c|c|c|c|c|}
\hline & & & & & Notat & & & & & \\
\hline & & & & & & & & & & \\
\hline & Lav & Per & Lav & Per & Lav & Per & Lav & Per & Lav & Per \\
\hline Lignées & & & & & & & & & & \\
\hline LFF07 & 0 & 0 & 0 & 0 & 0 & 5 & 5 & 12 & 5 & 22 \\
\hline LFF12 & 77 & 66 & 81 & 67 & 85 & 68 & 86 & 72 & 807 & 673 \\
\hline FPLATA & 0 & 0 & 0 & 1 & 28 & 45 & 40 & 63 & 96 & 155 \\
\hline
\end{tabular}

Tableau II. Répartition par classes des lignées testées au champ.

\begin{tabular}{|c|c|c|c|c|c|c|c|}
\hline \multirow[t]{2}{*}{ Lignées } & \multirow[t]{2}{*}{ Nombre d'observations } & \multicolumn{6}{|c|}{ Classes } \\
\hline & & $T S$ & $S$ & $T$ & $R$ & $H R$ & $?$ \\
\hline LFF07 & 7 & 0 & 0 & 0 & 5 & 2 & 0 \\
\hline LFF12 & 9 & 0 & 5 & 1 & 1 & 0 & 2 \\
\hline FPLATA & 11 & 1 & 1 & 0 & 2 & 3 & 4 \\
\hline
\end{tabular}

- la diminution du poids de 1000 grains $(-0,41 \%)^{\star \star}$;

- l'augmentation du poids spécifique $(+0,10 \%)$;

- la perte d'humidité $(-0,56 \%)$;

- la perte de rendement $(-7,10 \%)$ *.

La signification des notations est indiquée (NS, ${ }^{\star},{ }^{\star \star}$ ) au seuil $5 \%$.

Afin de classer notre matériel, les 3 notations hautement significatives $\left(^{\star \star}\right)$ ont été valorisées dans notre interprétation. Un coefficient leur a été attribué afin de juger de la résistance et de la sensibilité du matériel. Cette hiérarchisation des caractères nous a permis d'obtenir différentes "classes» dont les résultats obtenus sur les 650 lignées que nous avons étudiés sont les suivants : HR : hautement résistant (76) ; $\mathrm{R}$ : résistant (181) ; T- : tolérant (35) ; S- : sensible (200) ; TS- : très sensible (64) ; ?- : on ne peut pas conclure (94).

Le tableau II regroupe les résultats au champ des lignées dont nous avons présentés les résultats des observations en enceintes climatisées.

En affectant un coefficient de 1 pour les HR, 2 pour les R, 3 pour les T, 4 pour les $S, 5$ pour les
TS, nous obtenons les moyennes et variances suivantes (tableau III).

Dans la littérature, nous savons que B73 est considérée comme une lignée sensible, ce que confirment nos résultats.

D'après les résultats du disease index, nous savons que LFF07 peut être considérée comme une lignée résistante, nous avons ici 1,27< LFF07 <2,22.

FPLATA est classée résistante d'après les résultats du disease index. Au champ, nous obte-

Tableau III. Moyennes et variances obtenues après affectation d'un coefficient à chaque classe de résistance.

\begin{tabular}{lll} 
Lignées & Moyenne & Variance \\
\hline & & \\
B73 & 3,57 & 1,10 \\
LFF07 & 1,71 & 0,2 \\
LFF12 & 3,57 & 0,53 \\
FPLATA & 2,28 & 2,2 \\
\hline
\end{tabular}


Tableau IV. Ségrégation obtenue après recombinaison.

$\begin{array}{lc} & \text { avec symptômes } \\ \mathrm{R} \times \mathrm{R} & 6 \\ \mathrm{R} \times \mathrm{T} & 4 \\ \mathrm{R} \times \mathrm{S} & 14 \\ \mathrm{R} \times \mathrm{TS} & 30 \\ \mathrm{~T} \times \mathrm{S} & 198 \\ \mathrm{~S} \times \mathrm{S} & 284 \\ \mathrm{TS} \times \mathrm{TS} & 401 \\ & \\ \text { nons, } 0,79<\mathrm{FPLATA}<\mathrm{3}, 76 . \text { L'écart type est } \\ \text { trop important pour que nous puissions conclure. } \\ \text { Ceci est dû au fait que FPLATA extériorise les } \\ \text { symptômes à long terme (cf les résultats du } \\ \text { disease index). La notation faite au champ } 21 \mathrm{j} \\ \text { après l'inoculation n'est pas adaptée à cette } \\ \text { lignée. En effet, certaines années, des symp- } \\ \text { tômes sont visibles ; d'autres années, ils ne le } \\ \text { sont pas. }\end{array}$

LFF12 est sensible en enceintes climatisées ; au champ, nous avons 2,84 < LFF12 < 4,29, ce qui nous permet également de conclure à la sensibilité au champ.

\section{CONCLUSION}

L'objectif premier de ce travail était de classer les 650 lignées inoculées au champ. Dix notations nous ont permis de les regrouper en 5 classes.

Le deuxième consistait à mettre en évidence la corrélation entre les indications "champ" et les indications en «enceinte», ce qui a été démontré.

Les informations «champ» étant relativement difficiles à interpréter (plus de $14 \%$ des résultats sont inutilisables), et étant relativement onéreuses ( \pm 1000 francs par lignée), nous savons désormais que l'analyse en «enceinte» peut nous aider à choisir les lignées ayant un taux de résistance suffisant. Ces analyses peuvent être effectuées à n'importe quelle période de l'année et ne s'échelonnent que sur $37 \mathrm{j}$. L'avantage d'une telle méthode est un atout considérable pour le sélectionneur. inoculées

$\begin{array}{cc}1488 & 371: 1 \\ 1584 & 113: 1 \\ 1056 & 34: 1 \\ 1056 & 4: 1 \\ 1056 & 3: 1 \\ 1056 & 2: 1\end{array}$

\section{ÉTUDE DE LA GÉNÉTIQUE DE LA RÉSISTANCE}

Le troisième objectif avait pour but de choisir les meilleurs lignées, qui en recombinaison donneraient de nouvelles lignées présentant des résistances intéressantes pour la sélection. Une approche de la génétique de la résistance a donc été abordée. Des départs de sélection ont été inoculés et la ségrégation obtenue est la suivante (tableau IV) :

Cette ségrégation peut être comparée pour certains départs de sélection à ce qu'obtint Roane (Roane et al, 1983). Notre étude tend à montrer que la résistance est fortement dominante. Pour le sélectionneur, il suffit, dans son matériel de départ, de choisir au moins une lignée dont il connaît la résistance au MDMV-A pour avoir la certitude d'obtenir une lignée résistante à la souche analysée après une sélection classique, généalogique.

\section{RÉFÉRENCES}

Kuhn CW, Smith TH (1977) Effectiveness of a disease index system in evaluating corn for resistance to maize dwarf mosaic virus. Phytopathology 67, 288291

Roane CW, Tolin SA, Genter GF (1983) Inheritance of resistance to maize dwarf mosaic virus in maize inbred line Oh7B. Phytopathology 73, 845-850

Signoret PA, Caffier D, Quiot JB (1993) Geographical diversity of maize dwarf mosaic virus present in west Mediterranean area. Premier Colloque italofrançais de Phytopathologie, Algero-Sassari (Sardaigne), 14-18 avril 1993, Petria 3 (Sup 1), 78 